\title{
Parameter space exploration of bio-inspired hover kinematics
}

\author{
Ruben B.R. Vandenheede* \\ Delft University of Technology, Delft, Zuid-Holland, 2600AA, Netherlands \\ Luis P. Bernal ${ }^{\dagger}$ and Christian L. Morrison ${ }^{\ddagger}$ \\ University of Michigan, Ann Arbor, MI, 48105, United States \\ Sean Humbert ${ }^{\S}$ \\ University of Maryland, College Park, MD, 20742, United States
}

\begin{abstract}
An experimental investigation of the parameter space of biologically inspired hover kinematics for flapping wings is conducted. The present research continues earlier work conducted at a Reynolds number of $4.8 \times 10^{3}$ and with a reduced frequency of 0.38 . The kinematics considered are pitch and plunge motions based on the kinematics of the Agrius Convolvuli, which include a hover kinematic ignoring elevation angle, a hover kinematic incorporating a correction for elevation angle, and a harmonic pitch/plunge motion of the same amplitude. A parameter space exploration of these baseline motions is performed. First, the reduced frequency is increased to a value of 0.7 . Second, a phase lag of $10 \% \mathrm{~T}$ and $-10 \% \mathrm{~T}$ are introduced to study the effects of advanced and delayed rotation. Force measurements are reported and the results compared to the baseline motions. An increase in reduced frequency increases the average thrust coefficient substantially for all motions. The efficiency decreases slightly for all but the pure sinusoidal motion. Introducing a phase lag of $10 \%$ of the period changes the forces significantly. For delayed rotation thrust production and efficiency decrease by a large amount. Increases in thrust occur in the advanced rotation cases, although there is a penalty in efficiency.
\end{abstract}

\section{Nomenclature}

$A \quad$ Area swept by the wing, $\mathrm{m}^{2}$

$A R$ Aspect ratio, -

$C_{L} \quad$ Lateral force coefficient, -

$C_{T} \quad$ Thrust coefficient, -

$F_{\text {lateral }}$ Lateral force, $\mathrm{N}$

$L_{\text {ref }}$ Reference length

$M \quad$ Figure of merit, -

$M_{x} \quad$ Moment around the axis $\mathrm{x}, \mathrm{Nm}$

$P \quad$ Power, $\mathrm{W}$

$R \quad$ Semi-span, $m$

Re Reynold number

$T$ Thrust force, $\mathrm{N}$

$U$ Velocity

$\Phi \quad$ Flapping amplitude, rad

$c \quad$ Mean chord length, $\mathrm{m}$

\footnotetext{
*Graduate Research Assistant, Department of Aerospace Engineering, vdh.ruben@gmail.com

${ }^{\dagger}$ Associate Professor, Department of Aerospace Engineering, lpb@umich.edu

${ }^{\ddagger}$ Graduate Research Assistant, Department of Aerospace Engineering, cmorrisz@umich.edu

$\S$ Assistant Professor, Department of Aerospace Engineering, humbert@umd.edu
} 
$f \quad$ Flapping frequency, $\mathrm{Hz}$

$h \quad$ Plunge position experiment

$h_{0} \quad$ Plunge amplitude experiment

$k \quad$ Reduced frequency

$\alpha \quad$ Feathering angle, rad

$\delta \quad$ Pitch angle, rad

$\nu_{a} \quad$ Viscosity of air

$\nu_{w} \quad$ Viscosity of water

$\phi \quad$ Flapping angle

$\rho \quad$ Density

$\theta \quad$ Elevation angle

- Denotes the $1^{\text {st }}$ time derivative

- Denotes an averaged quantity

\section{Introduction}

Flapping wings have unique characteristics that can be exploited in the development of micro air vehicles, for example flapping wings perform very well in confined spaces, they could be very small and have a low energy consumption. Applications can range from reconnaissance to search and rescue to espionage. Small birds and insects display these flight characteristics which has motivated extensive research of the aerodynamics of bio-inspired flapping wing kinematics by biologist and engineers. A very well studied example of a biological flapping flyer is the hawkmoth. This moth is an excellent study object for a number of reasons. ${ }^{4}$ The wing beat of the moths is strikingly consistent. This consistent wing beat holds for an individual moth as well as between different moths. The main features dominant in insect flapping flight are present in the stroke of the hawkmoth, without the complications of extra mechanisms present in a number of other insects, e.g. clap \& fling mechanisms and exaggerated ventral flexion.

In this paper the parameter space of the kinematics, inspired on the hover kinematics of the hawkmoth, is experimentally investigated. The experiments for the baseline motions were initially conducted at the Reynolds number and reduced frequency of the hawkmoth in hover, i.e. $4.8 \times 10^{3}$ and 0.38 respectively. The parameter space is now expanded to a higher reduced frequency and the introduction of advanced and delayed rotation.

\section{Experimental setup}

The experiments are performed in The University of Michigan free surface water channel. The circulating channel has total volume of about 8,000 gallons, and can be seen in Figure 1. The test section is made out of transparent float glass, measures 610 by $610 \mathrm{~mm}$, and is $2.44 \mathrm{~m}$ long. The transparent test section walls facilitate flow visualization and particle image velocimetry experiments. The channel is capable of producing very low turbulence flow, the free turbulence intensity for the possible flow speeds is always less than $1 \%$ of the freestream velocity, measured at various flow speeds with Particle Image Velocimetry (PIV). Flow speeds can be varied from 5 to $40 \mathrm{~cm} / \mathrm{s}$. For this experimental campaign there is no flow in the channel, as we are investigating hover cases.

A Zimmerman wing is used in all experiments; the wing has a chord of $79.4 \mathrm{~mm}$ and a span of $241.3 \mathrm{~mm}$. The planform shape consists of two ellipses joined at the quarter chord of the wing. The major axes are equal to the span. The minor axes are equal to one quarter and three quarters of the wing chord respectively, cf. Figure 2a. The wing planform is laser-cut (PLS6.75, Universal Laser Systems) out of a transparent acrylic plate with a thickness of $2.75 \mathrm{~mm}$. No post-processing is applied and thus the edges of the wing are sharp right angles. The thickness of the wing, $2.75 \mathrm{~mm}$, is substantial and the wing is henceforth assumed to be rigid; during the experiments no deformation of the wing was observed.

The wing is clamped and mounted on a rig with two motors, hanging vertically in the water channel, 


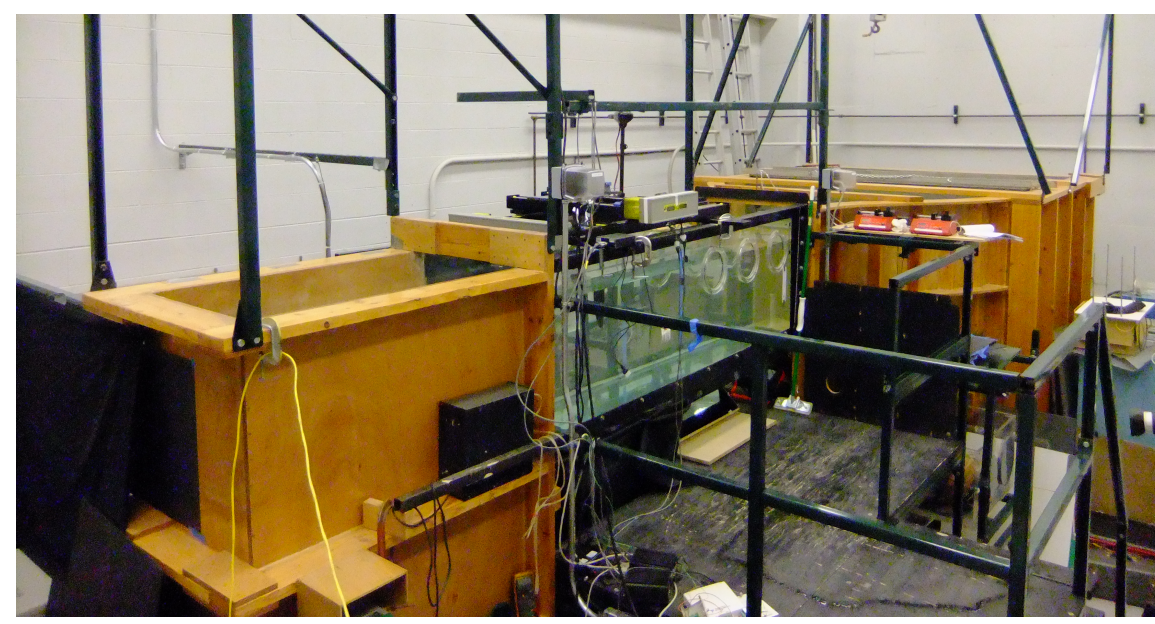

Figure 1: Overview of the University of Michigan water channel

as shown in Figure 2b. The motors are capable of performing pitch-plunge motions with a high degree of accuracy as discussed in the next section.

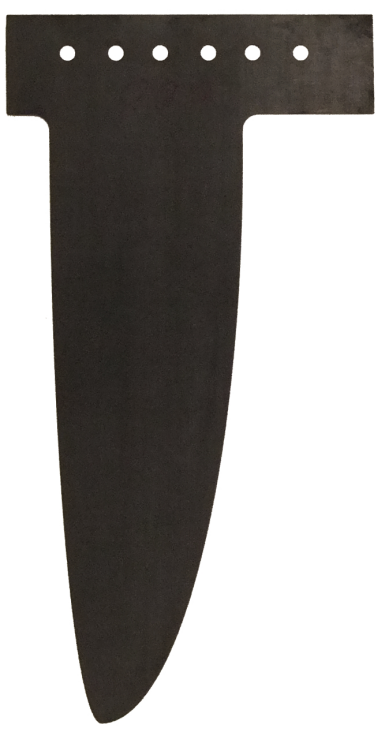

(a) Wing planform

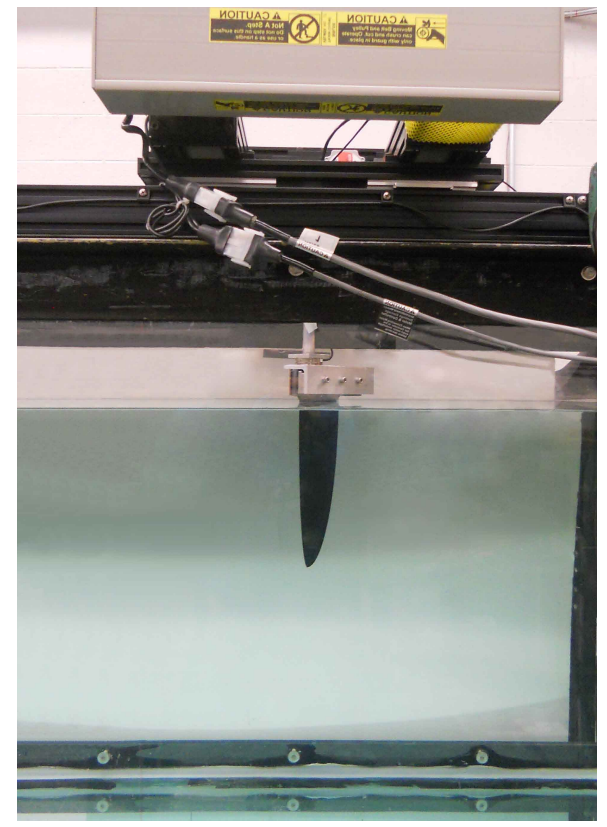

(b) Wing vertically mounted in the water channel

Figure 2: Experimental Setup

\section{Determining the relevant scaling and non-dimensional parameters}

The hover kinematics are inspired by a hawkmoth, the Agrius Convolvuli. As a starting point for determining the relevant scaling and non-dimensional parameters, the values of the Agrius Convolvuli are used. 


\section{A. Hawkmoth, Agrius Convolvuli}

The Agrius Convolvuli is a subspecies of the hawkmoth, Sphingidae. It is a large species with a wingspan of about $80-105 \mathrm{~mm}$. Similarly to hummingbirds, they have evolved to feed on nectar while hovering in front of a flower. This capability makes them an excellent study object for flapping hovering flight.

The kinematics of this moth in hovering flight are well known, cf. Section IV. The motion is relatively simple and does not include a clap and fling mechanism. The Agrius Convolvuli is four-winged, but the motion of the wings on both sides in hover is nearly analogues and can be assumed to act as one wing. The wing planform of both wings together is similar to the Zimmerman planform and will be modeled as such.

The parameters determining the dynamics are presented in Table 1 . The flapping frequency and amplitude are those of the moth in hover.

Table 1: Agrius Convolvuli parameters in hover and experiment parameters

\begin{tabular}{l|l|l}
\hline Parameter & $\begin{array}{l}\text { Value Agrius } \\
\text { convolvuli }\end{array}$ & $\begin{array}{l}\text { Value } \\
\text { Experiment }\end{array}$ \\
\hline Mean chord length $c$ & $18.3 \mathrm{~mm}$ & $62.4 \mathrm{~mm}$ \\
Semi-Span $R$ & $50.5 \mathrm{~mm}$ & $241.3 \mathrm{~mm}$ \\
Aspect ratio $A R$ & 2.76 & 3.87 \\
Flapping frequency & $26.1 \mathrm{~Hz}$ & $0.15 \mathrm{~Hz}$ \\
Flapping/Plunging & $2 \mathrm{rad}$ & $129.1 \mathrm{~mm}$ \\
amplitude $\Phi / \mathrm{h}$ & & \\
\hline
\end{tabular}

In order to calculate the relevant dimensionless parameters for hover, a reference point on the wing needs to be chosen. In general the wing tip is used for this purpose, ${ }^{2}$ however since the objective is to capture the most relevant phenomena and try to represent these in a pitch-plunge motion, the tip velocity will make the scaling parameters too large. A reasonable approximation is to use three quarters of the span $\left(L_{r e f}=0.75 R_{m}\right)$.

$$
U_{\text {ref }}=2 \Phi f L_{r e f}
$$

The Reynolds number can then be rewritten as follows.

$$
R e=\frac{U_{r e f} c_{m}}{\nu_{a}}=\frac{2 \Phi f_{m} L_{r e f} c_{m}}{\nu_{a}}
$$

In turn the reduced frequency, equation 3 , is based on the same reference velocity.

$$
k=\frac{\pi f_{m} c_{m}}{U_{r e f}}
$$

With the reference velocity based on three quarters of the span the Reynolds number and reduced frequency are $4.8 \times 10^{3}$ and 0.38 respectively.

\section{B. Relevant parameters for the experiment}

The experiment is a pitch-plunge motion, hence the reference velocity is equal to the average velocity during half of the period, cf. equation 4, depending only on the amplitude and frequency of the motion.

$$
U_{\text {ref }}=4 f h_{0}
$$

Substitution of the reference velocity, equation 4, leads to the following expression for the reduced frequency of the experiment. Note that in the pitch-plunge case, for a certain wing, the reduced frequency is solely dependent on the amplitude of the motion.

$$
k=\frac{\pi c}{4} \frac{1}{h_{0}}
$$

$$
4 \text { of } 20
$$


Similarly the Reynolds number can be written as.

$$
R e=\frac{4 c}{\nu_{w}} f h_{0}
$$

In these experiments the Reynolds number is kept constant at the value of the hawkmoth, i.e. $4.8 \times 10^{3}$. The reduced frequency is kept at 0.38 for the delayed and advanced rotation cases and to investigate the effect of an increase in reduced frequency to 0.7 .

\section{Hover kinematics}

Three motions are considered in this study, the first hover motion ignores the loss of elevation angle and simply converts the flapping and feathering angle, as shown in Figure 3a. This motion will be referred to as hover motion 1 or HM1.

The second hover motion, Figure 3b, incorporates a correction in the flapping and feathering angle for the elevation angle. This motion will be referred to as hover motion 2 or HM2. The correction has an effect on both the pitch and plunging motion. In comparison to the pitch motions, the plunging motions is hardly altered. The difference between HM1 and HM2 is thus dominated by the pitch angle.

The third motion is a pure sinusoidal motion with the same maximum pitch and plunge amplitudes as the basic Agrius Convolvuli kinematics. The sinusoidal kinematics are plotted in Figure 3c.

\section{Force data acquisition}

\section{A. Data acquisition process}

Per motion, force data is acquired in 6 separate sets of 25 cycles. The first five cycles are cropped from each measurement eliminating any start up phenomena that might occur. The data is then calibrated and converted to thrust and lateral force components. The thrust is defined as the force perpendicular to the plunge motion, similarly the lateral force is the force lateral to the plunge motion.

The thrust and lateral force components are filtered with a low pass Butterworth filter with a cut off frequency of $3 \mathrm{~Hz}$. To eliminate the phase shift introduced by the filter, it is run over the data in the forward and reverse time direction using the MATLAB filtfilt function. This process ensures zero phase distortion and squares the magnitude of the original filter.

Next the remaining 120 cycles are phase averaged and the sample mean and the corresponding standard deviation are calculated.

\section{B. Non-dimensionalization of the data}

The resulting force data are non-dimensionalized by the dynamic pressure (based on the reference velocity $U_{\text {ref }}$ equal to $4 f h_{0}$, Section III B) and the projected wing surface area. The thrust coefficient is then defined as shown in equation 7 .

$$
C_{T}=\frac{T}{q S}
$$

Similarly the lateral force coefficient is normalized by the same dynamic pressure and the projected wing surface area.

To quantify the efficiency of thrust production, the ideal power is calculated using simple momentum theory. ${ }^{1}$ 


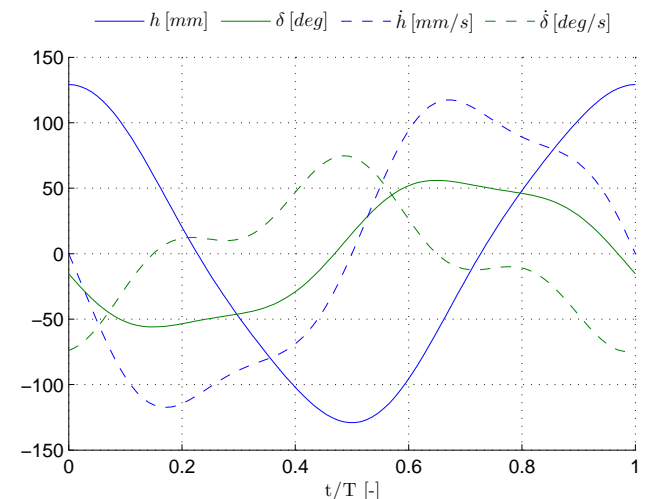

(a) Pitch-plunge hover motion experiment ignoring the loss of elevation angle (hover motion 1)

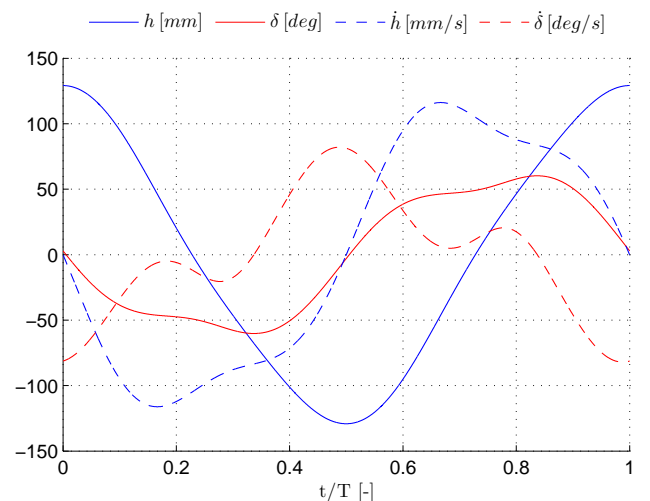

(b) Pitch-plunge hover motion experiment with compensation for the loss of elevation angle (hover motion 2)

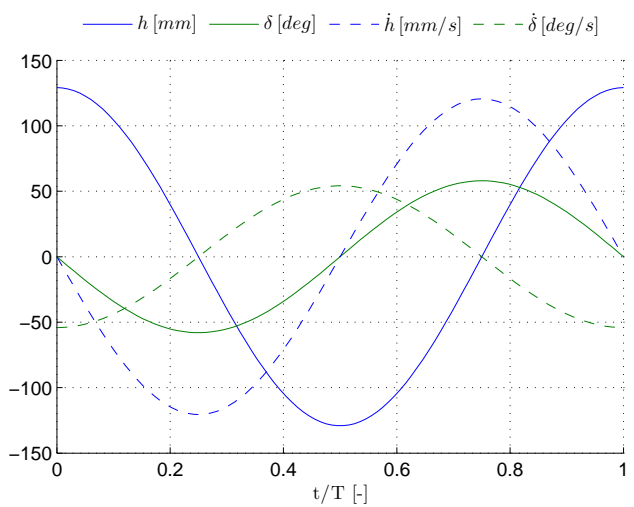

(c) Pure Sinusoidal pitch-plunge motion with the same amplitude and frequency as the Agrius Convolvuli

Figure 3: Resulting kinematics pitch-plunge kinematics of the experiments

$$
P_{\text {ideal }}=\sqrt{\frac{T^{3}}{2 \rho A}}
$$

Where $T$ is the average thrust produced, $\rho$ is the density of the fluid and $A$ is the area swept by the wing. The power input is calculated from the force and moment measurements by multiplying the angular speed by the corresponding moment and the translational velocity by the corresponding force, as shown in equation 9 .

$$
P=F_{\text {lateral }} \dot{h}+M_{z} \dot{\delta}
$$

A convenient figure of merit, $M$, extensively used in helicopter hover analyses is then defined as follows.

$$
M \equiv \frac{P_{\text {ideal }}}{P}
$$

\section{Effects of Changes in Phase}

The parameter space is first expanded by introducing phase lag. The phase lag of the pitch angle is expressed in percentages of the period. Two different cases have been added to the baseline motions, $\pm 10 \% T$. 


\section{A. Force coefficients}

The results for HM1 are shown in Figure 4 and Table 2. The delayed rotation case, i.e. having a phase lag of $-10 \% T$, produces a lot less thrust than the baseline case. Furthermore the figure of merit drops to a value of only 0.23 . The opposite is true for the advanced rotation case, the thrust is $34 \%$ higher than the baseline case with a decrease in the figure of merit from 0.47 to 0.41 . In both cases there is a shift in the location of the minimum corresponding approximately to the amount of phase lag for the advanced rotation case, and less then half of the amount of phase lag for the delayed rotation case. For all three motions the minima are close to zero compared to the maxima of the motions.

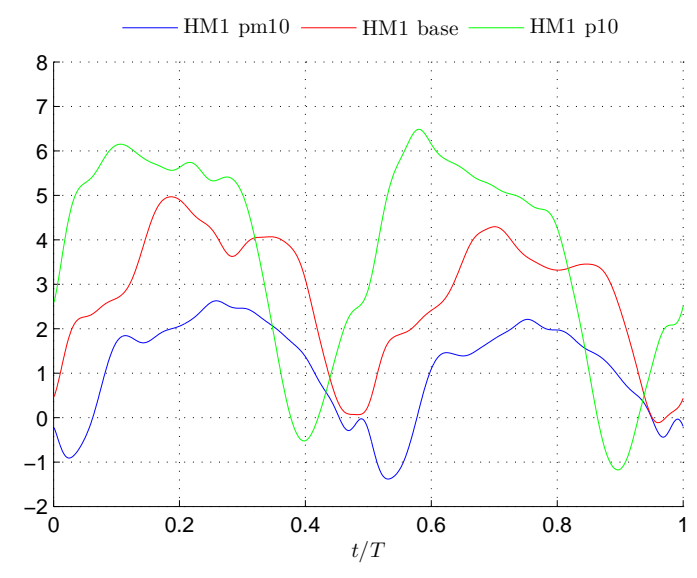

(a) Thrust coefficient

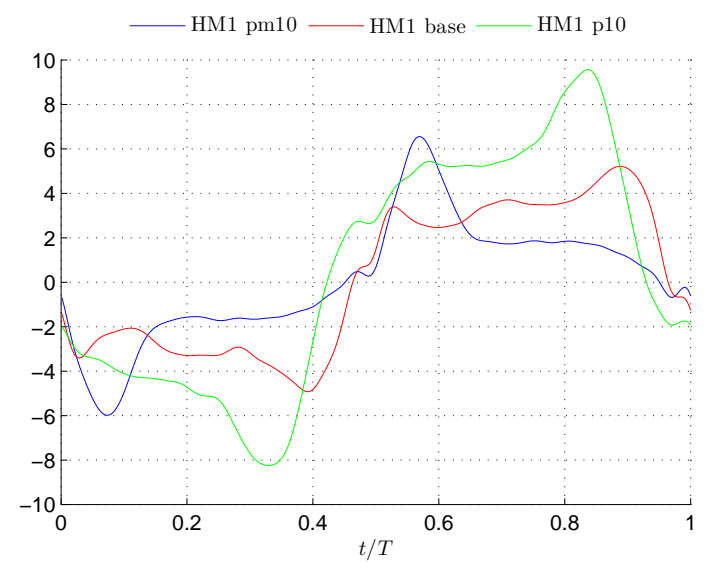

(b) Lateral force coefficient

Figure 4: Force coefficient for HM1 with with phase lag

A visual representation of the force vectors on the airfoil is shown in Figure 5. The maximum force builds up rapidly for the advanced rotation case and remains high throughout the stroke until the airfoil rotates and the orientation of the force vector becomes unfavorable. The pattern for the baseline case is similar but less aggressive. For the advanced rotation case the forces are smaller than the two other cases and the orientation at maximum force is less favorable.

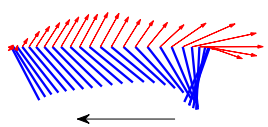

(a) HM1 $-10 \% T$

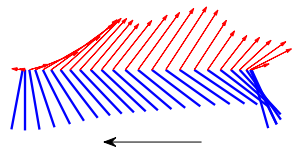

(b) HM1 baseline motion

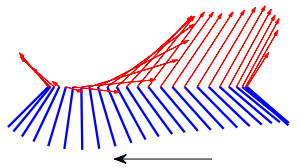

(c) $\mathrm{HM} 1+10 \% \mathrm{~T}$

Figure 5: Visualization of HM1 with phase lag and the generated forces

Similarly, for the harmonic motion (Table 3), the delayed rotation case produces less than half of the thrust and has a figure of merit of only 0.17 . The advanced rotation case however produces a huge amount of thrust more compared to the baseline case, about $47 \%$ more thrust. The thrust and lateral force coefficients are plotted in Figure 6. For the delayed rotation case the minimum has shifted by less than half the amount of phase lag, i.e. less than $5 \% T$ and for the advanced rotation the shift is roughly equal to the phase lag. 
Table 2: HM1 force coefficients for different phase lag

\begin{tabular}{c|ccc}
\hline & HM1 $-10 \% T$ & HM1 & HM1 $+10 \% T$ \\
\hline$C_{T}$ & 1.11 & 2.79 & 3.74 \\
$\left|C_{\text {Lateral }}\right|$ & 2.17 & 3.08 & 4.81 \\
$M$ & 0.23 & 0.47 & 0.41 \\
\hline
\end{tabular}

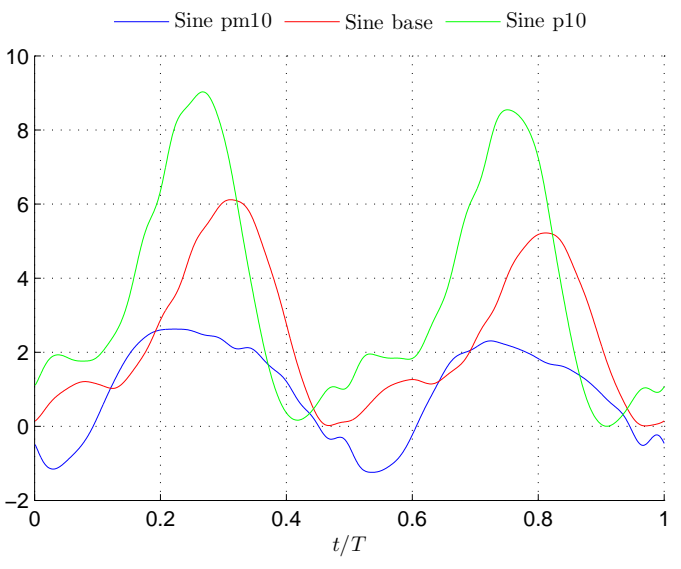

(a) Thrust coefficient

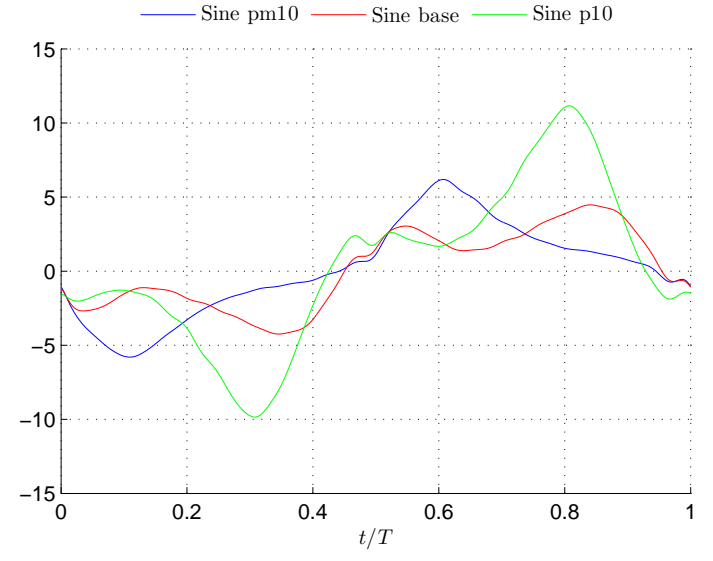

(b) Lateral force coefficient

Figure 6: Force coefficients for the purely sinusoidal motion with phase lag of $\pm 10 \%$

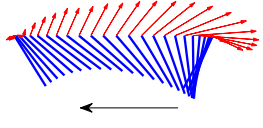

(a) sine $-10 \% \mathrm{~T}$

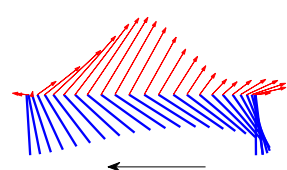

(b) sine baseline motion

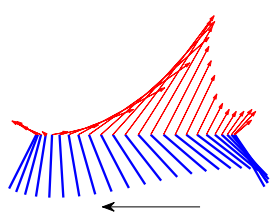

(c) sine $+10 \% \mathrm{~T}$

Figure 7: Visualization of sine with phase lag and the generated forces

For both HM1 and harmonic motion the thrust production is greatly enhanced by advanced rotation. The efficiency however decreases from 0.47 to 0.41 and from 0.49 to 0.39 respectively. The location of the maximum force shifts by approximately the amount of phase lag for the advanced rotation cases. The delayed rotation is detrimental for both thrust and efficiency in both motions.

\section{B. Dye flow visualization}

At $0.0 t^{*}$ the HM1 delayed rotation case starts out with a cloud of dye on top of the airfoil. A small vortex forms at both the LE and the TE as the airfoil translates. The vortices are shed immediately, the topology is sketched in Figure 8a. At this point the normal force has dropped to a relatively constant value and as the airfoil translates it seems to be fully stalled and no formation of vortices was observed in the flow 
Table 3: Sine force coefficients for different cases of phase lag

\begin{tabular}{c|ccc}
\hline & Sine $-10 \%$ & Sine & Sine $+10 \%$ \\
\hline$C_{T}$ & 0.96 & 2.39 & 3.51 \\
$\left|C_{\text {Lateral }}\right|$ & 2.53 & 2.39 & 4.21 \\
$M$ & 0.17 & 0.49 & 0.39 \\
\hline
\end{tabular}

visualization data, except at the tip. A tip vortex is present during the stroke, the direction of rotation is counter clockwise for an observer looking parallel with the plunge motion and the downstream direction on the left. The distance between the shed tip vortices in the wake varies between one and two chord lengths in downstream direction depending on the stroke. The average spanwise flow component from the tip to the three quarter chord position was $0.65 U_{\text {ref }}$.

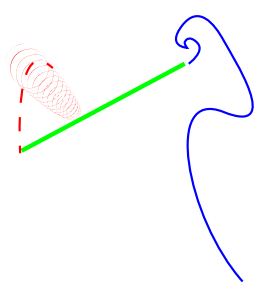

(a) $0.14 t^{*}$

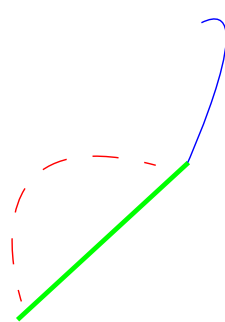

(b) $0.42 t^{*}$

Figure 8: Sketches of the flow visualization features HM1 with a $10 \% T$ delayed rotation

The motion is not efficient because it is never able to form a proper LEV during the stroke, furthermore the force peak occurs when the airfoil is perpendicular to the plunge direction and hence in the buildup this force causes some negative thrust.

For the HM1 advanced rotation case a TE starting vortex is created at the very start of the stroke. There is evidence of an LEV forming as sketched in Figure $9 \mathrm{~b}$ and a tip vortex seems to be present early in the stroke. With the advent of a new TEV due to the rotation at $0.27 t^{*}$ the tip vortex seems to disappear. The LEV grows very large in size, cf. Figure 9c. The normal force changes sign after the change in sign of the pitch motion and hence the thrust is negative from 0.38 to $0.42 t^{*}$. The average spanwise flow velocity measured $1.16 U_{\text {ref }}$. Furthermore a large spanwise contraction of the wake was observed. The contraction was difficult to quantify due to the diffusion of the dye, and it is estimated that the wake had contracted by roughly 2 chord lengths at 2 chord lengths downstream.

The motion seems to be able to create large forces, with the orientation of these forces in a favorable direction. A gain of $34 \%$ thrust compared to the baseline motion with a decrease in efficiency of $13 \%$.

A vortex is created at the leading edge and the trailing edge early in the stroke of the purely sinusoidal delayed rotation case, Figure 10a. Both vortices are shed immediately and a tip vortex is formed simultaneously. As the stroke progresses the tip vortex becomes more pronounced and no LEV was visible. At stroke reversal a small TEV is formed due to the rotation of the wing and rapidly broken up before the stroke actually reverses direction. Average spanwise flow speeds were measured to be up to $0.56 U_{\text {ref }}$.

No evidence of high lift mechanisms was observed in this stroke. The airfoils seems to be stalled throughout the entire stroke.

The sinusoidal advanced rotation case generates a faint TE starting vortex as it begins to translate. The 


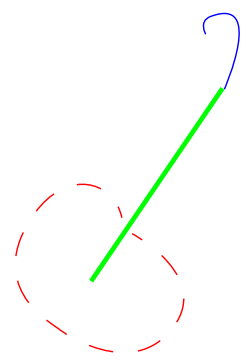

(a) $0.07 t^{*}$

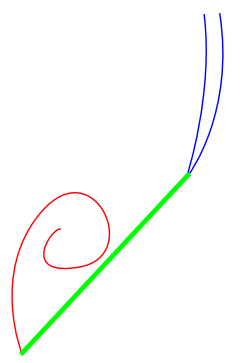

(b) $0.2 t^{*}$

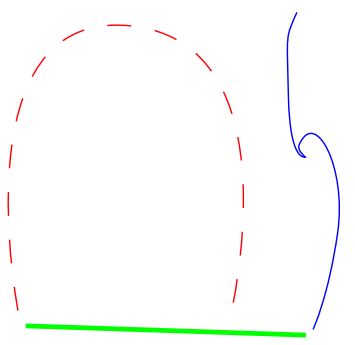

(c) $0.38 t^{*}$

Figure 9: Sketches of the flow visualization features HM1 with a $10 \% T$ advanced rotation

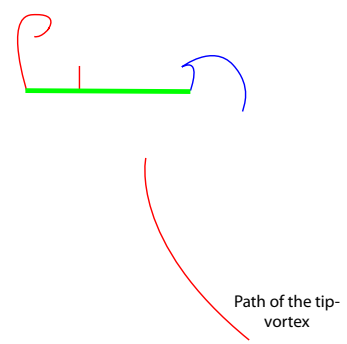

(a) $0.11 t^{*}$

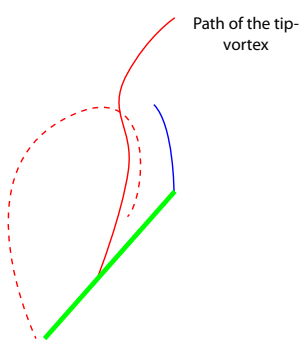

(b) $0.43 t^{*}$

Figure 10: Sketches of the flow visualization features sinusoidal motion with a $10 \% T$ delayed rotation

first evidence of the LEV is seen at $0.24 t^{*}$, and the LEV keeps growing until the end of the stroke. A peak in normal force was found just before $0.3 t^{*}$ and the flow topology at this point is sketched in Figure 11b. In this Figure a distinct LEV and tip vortex are depicted.

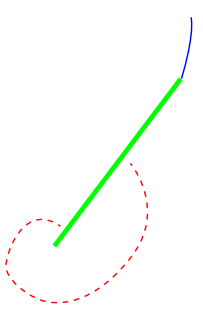

(a) $0.1 t^{*}$

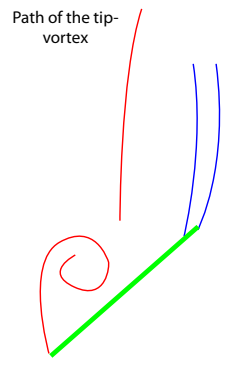

(b) $0.3 t^{*}$

Figure 11: Sketches of the flow visualization features sinusoidal motion with a $10 \% T$ delayed rotation

The motion is $47 \%$ more effective in the production of thrust compared to the baseline motion. The increase in thrust comes at the price of a reduction of $20 \%$ in efficiency.

For both motions the flow topology is comparable to the baseline motions for the advanced rotation case. The time history of the force coefficients has roughly the same shape, only more pronounced. Conversely the delayed rotation cases do not seem to generate high lift mechanisms in the flow and the peaks in the generated force occur at unfavorable orientations of the airfoil. Incorporation of advanced and delayed rotation can increase and decrease thrust significantly, up to $60 \%$ difference in the thrust force with a delay 
in the rotation of only $10 \% \mathrm{~T}$. Hence these mechanisms might be used to generate large forces used for quick maneuvres in exchange for efficiency.

\section{Effects of Changes in Reduced Frequency}

The reduced frequency provides a measure of the unsteadiness associated with a flapping wing. ${ }^{2}$ As an expansion of the parameter space the reduced frequency is increased from 0.38 to 0.7 .

\section{A. Force coefficients}

In Figure 12, the resulting force coefficients are plotted. Here all motions are plotted in the same plot, a plot of each individual motion at a reduced frequency of 0.38 and 0.7 can be found in Appendix A. It can be seen that the thrust coefficient for a reduced frequency of 0.7 is slightly lower in the first half stroke as compared to the values with a reduced frequency of 0.38 . However in the second half stroke the thrust is considerably larger, making up entirely for the loss in the first half stroke. That is for all motions the average thrust coefficient (Table 4) is larger for the largest reduced frequency. The increase in reduced frequency amounts to an increase in thrust coefficient of 12 to $18 \%$. All the while the figure of merit does not vary significantly. Note that the Sine motion is the only motion for which the figure of merit increases.

The general trends of the baseline motions do not change, HM1 still produces the largest amount of thrust, followed by HM2 and the sine motion. The sine motions produces the highest measured thrust force coefficient for the highest efficiency at the reduced frequency of $k=0.7$.

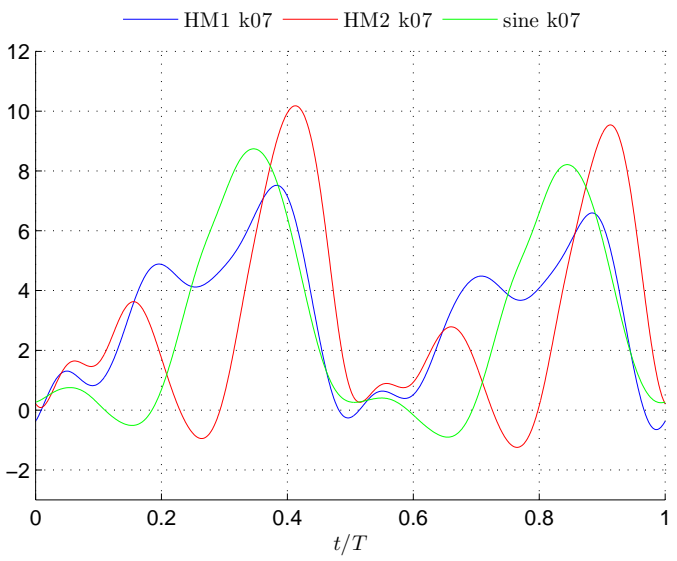

(a) Thrust coefficient

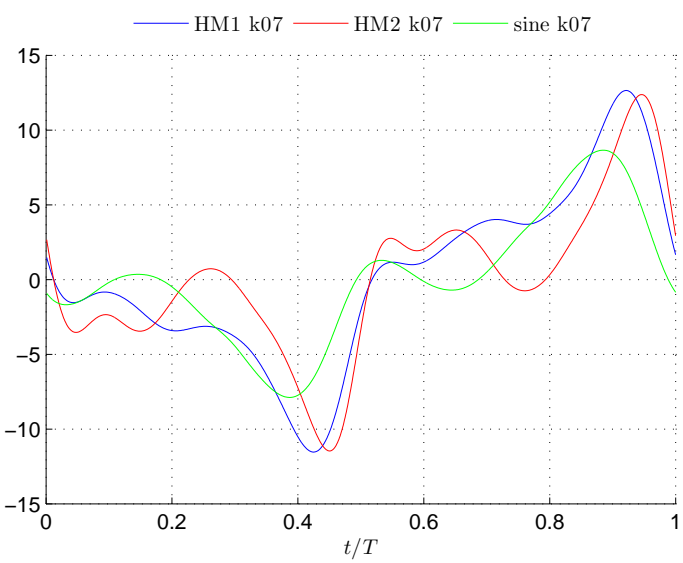

(b) Lateral force coefficient

Figure 12: Force coefficient for motions with a reduced frequency of 0.7

Table 4: Motion force coefficients

\begin{tabular}{c|cc|cc|cc}
\hline & \multicolumn{2}{|c|}{ HM1 } & \multicolumn{2}{c|}{ HM2 } & \multicolumn{2}{c}{ Sine } \\
& $k=0.38$ & $k=0.7$ & $k=0.38$ & $k=0.7$ & $k=0.38$ & $k=0.7$ \\
\hline$C_{T}$ & 2.79 & 3.22 & 2.64 & 2.95 & 2.39 & 2.81 \\
$\left|C_{\text {Lateral }}\right|$ & 3.00 & 4.61 & 2.98 & 3.73 & 2.39 & 3.05 \\
$M$ & 0.47 & 0.42 & 0.48 & 0.46 & 0.49 & 0.51 \\
\hline
\end{tabular}




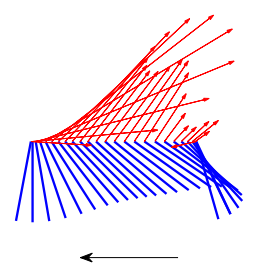

(a) HM1

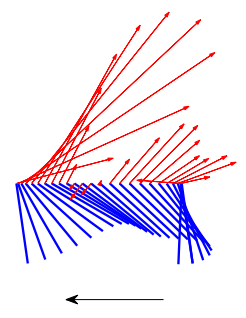

(b) HM2

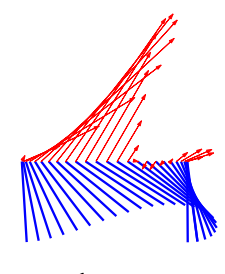

(c) Sine

Figure 13: Visualization of HM1 at a reduced frequency of 0.7 and the generated forces

\section{B. Dye flow visualization}

At the higher reduced frequencies the flow topology is rather similar compared to the baseline motions.

The stroke for HM1 starts out with the presence of the LEV from the previous stroke, Figure 14a. A starting vortex appears at the trailing edge and a small tip vortex is visible at $0.09 t^{*}$. The formation of an LEV is estimated to start at $0.2 t^{*}$, at this point the tip vortex is clearly visible. From the side view it is deduced that the average spanwise flow from the tip to the three quarter span position is in the order of $1 U_{\text {ref }}$ from 0.09 to $0.3 t^{*}$. Spanwise flow was observed at the three quarter span position and the half span position as well.

Figure 14c depicts a sketch of the flow at $0.46 t^{*}$. The leading edge has grown somewhat larger at this point and the trailing edge dye at half span is further downstream than at the three quarter span position. The tip vortex seems to remain strong up to the very end of the stroke. The TEV formed was formed at approximately $0.38 t^{*}$. At stroke reversal, the tip vortex fades out.

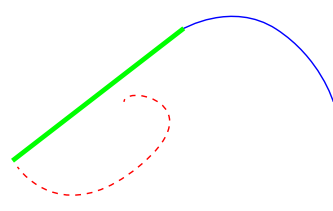

(a) $0.05 t^{*}$

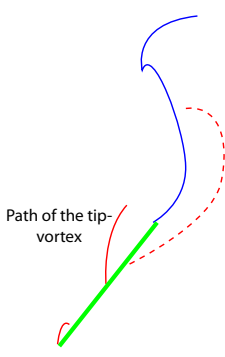

(b) $0.2 t^{*}$

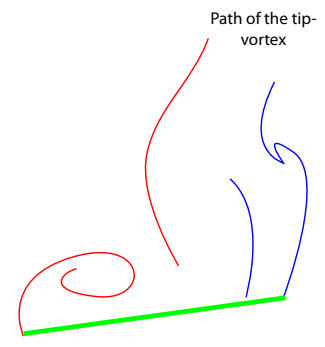

(c) $0.46 t^{*}$

Figure 14: Sketches of the flow visualization features HM1 with a reduced frequency of 0.7

HM2 starts with a similar flow picture as its baseline motion, Figure 15c; the presence of a LEV from the previous stroke during the supination is clearly visible. The same holds for the trailing edge vortex originating from the early supination phase, approximately $-0.12 t^{*}$. The trailing edge vortex is located further downstream at the half span position. A diffusive cloud of red dye is seen between the LEV and TEV, this cloud is the burst LEV formed earlier during the stroke. The wing starts translating faster and a starting vortex forms at the trailing edge. As the stroke progresses a small LEV is formed at roughly $0.16 t^{*}$, sketched in Figure 15a, and seems to detach at $0.3 t^{*}$. At the start of the supination a trailing edge vortex is formed as the LEV breaks down further. A new LEV is formed at $0.45 t^{*}$ due to the rotation of the airfoil.

The sinusoidal motion has the simplest flow topology with its main features sketched in Figure 16. The LEV from the previous stroke is still present at the beginning of the stroke and gets convected downstream 


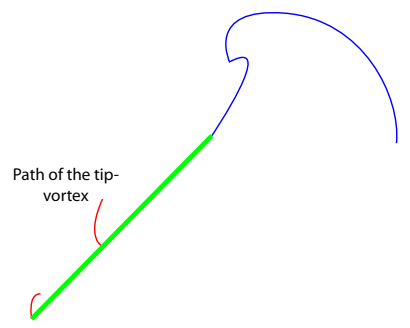

(a) $0.16 t^{*}$

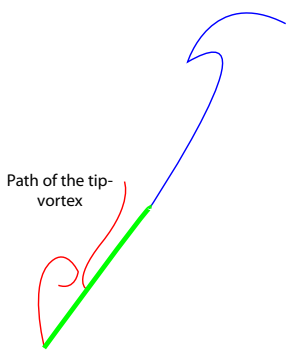

(b) $0.26 t^{*}$

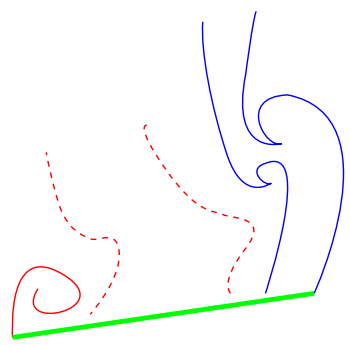

(c) $0.5 t^{*}$

Figure 15: Sketches of the flow visualization features HM2 with a reduced frequency of 0.7

as the stroke progresses. A streak line from the tip is visible at $0.15 t^{*}$, this could potentially be a tip vortex. Spanwise flow was observed originating at the tip of the airfoil and spreading to a region over half of the span by the end of the stroke. The average spanwise flow from the tip to the quarter span position between 0.12 and $0.38 t^{*}$ was measured to be $0.82 U_{\text {ref }}$. The first indications of a LEV in the flow become visible at $0.3 t^{*}$. The LEV develops further till end of the stroke, at which point it gets convected downstream.

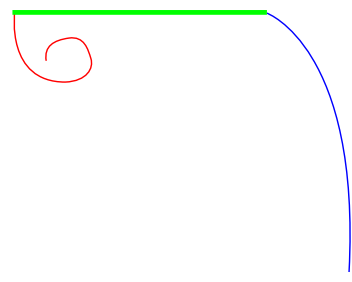

(a) $0.0 t^{*}$

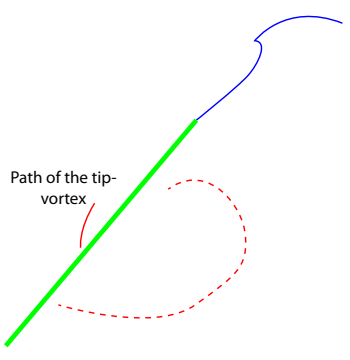

(b) $0.15 t^{*}$

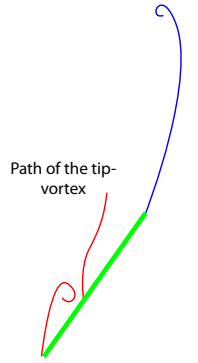

(c) $0.3 t^{*}$

Figure 16: Sketches of the flow visualization features sine with a reduced frequency of 0.7

The structure of the wake seems fairly simple compared to HM1 and HM2, and can be described as a vortex street with one shed vortex per stroke. As seen from the side view, there seems to be a large contraction of the wake in spanwise direction, it is estimated that the wake is about one chord length high at about four chord lengths downstream from the trailing edge of the airfoil.

Generally at the higher reduced frequency the LEV seems to be closer tied to the the airfoil compared to the baseline motion and the vortices seem stronger in strength. In the baseline motion there is no distinct tip vortex observed using the flow visualization technique. Conversely in the higher reduced frequency cases there are some streak lines originating from the tip that are strong indications of a tip vortex. At stroke reversal the flow moves into its own wake, in these cases, $k=0.7$, the leading edge vortex seems to keep rotating longer and perhaps until it joins the TEV, as opposed to the LEV stopping to rotate almost instantly at stroke reversal. In the baseline motions the dye cloud originating from the LEV of the previous stroke seems to be stationary.

The spanwise flow is higher, and remains in the same order of magnitude compared to the baseline motions. The contraction of the wake in spanwise direction is larger compared to the baseline motions, an indication that higher thrust forces are produced.

The flow field is comparable to that of the baseline motion. The differences are mainly that the motions are more aggressive and vortices in the flow field interact more with one another since they are around for 
a longer portion of the period.

\section{Conclusions and Recommendations}

In this paper the parameter space of the pitch-plunge motions, presented in 'Force generation of bioinspired hover kinematics', ${ }^{3}$ is explored. The parameter space is expanded in two dimensions, i.e. increasing the reduced frequency and introducing delayed and advanced rotation.

The first motion, HM1, ignores the elevation angle and simply translates the flapping angle into a plunge motion by using a reference speed at $75 \%$ of the span and uses the feathering angle as the pitch angle. The second motion is similar but encorporates a correction for the loss of elevation angle by adding the elevation angle to the feathering angle as an effective angle of attack. A third motion is a pure sinusoidal motion with the same Reynolds number and reduced frequency, i.e. $4.8 \times 10^{3}$ and 0.38 respectively (cf. Agrius Convolvuli). The thrust production and its efficiency are measured with a ATI Mini40 force sensor. A dataset is obtained over 120 cycles, filtered with a Butterworth filter (cut-off frequency of $3 \mathrm{~Hz}$ ) and phase averaged.

For the baseline motions HM1, HM2 and Sine the average thrust coefficients are 2.79, 2.64 and 2.39 respectively. The efficiencies are all in the same order of magnitude, roughly 0.48 .

A phase lag of plus and minus $10 \%$ of the period was introduced. In the delayed rotation cases there were no high lift vortex mechanisms observed in the flow and the peaks in the force did not occur at a favorable orientation for thrust generation. The thrust produced was $60 \%$ less compared to the baseline kinematics. Therefore the efficiency of the delayed rotation cases dropped dramatically. The introduction of advanced rotation yields an increase in thrust of 34 to $47 \%$ with a reduction of up to $20 \%$ in efficiency. The flow topology was remarkably similar to the baseline kinematics with a slight shift in phase. The application of both advanced and delayed rotation can potentially provide a mechanism to create large differences in forces for maneuverability of flapping wing flyers.

Further expansion of the parameter space was done by increasing the reduced frequency from 0.38 to 0.70. Increasing the reduced frequency yields an increase in thrust of 12 to $18 \%$ with a change in efficiency of $-11,-4$, and $+4 \%$ for HM1, HM2 and the sine motion respectively. Note that for the purely harmonic case the efficiency increases with increasing reduced frequency. It might be favorable for roboflyers to design kinematics at higher reduced frequencies for certain kinematics to attain a higher thrust and effciency in hover. Overall similar flow structures to the baseline cases were observed in the higher reduced frequency cases.

The expansion of the parameter space was performed in terms of reduced frequency and phase lag. It was found that both affect the forces and the efficiency of the motion. In the present study vortex strength was not characterized and it remains an open question whether an increase in force generation is linked to the vortex strength or if it is due to an increase in non-circulatory effects, especially in the high reduced frequency case. When clarity is found on this issue it would also be beneficial to investigate the variation of multiple parameters together and characterize the inter-parameter dependency.

\section{Acknowledgments}

This work has been supported in part by the Air Force Office of Scientific Research's Multidisciplinary University Research Initiative.

\section{References}

\footnotetext{
${ }^{1}$ Barnes W. McCormick. Aerodynamics, Aeronautics, and Flight Mechanics. Wiley, 1994.

${ }^{2}$ W. Shyy, H. Aono, S.K. Chimakurthi, P. Trizila, C.-K Kang, C.E.S. Cesnik, and H. Liu. Recent progress in flapping wing
} 
aerodynamics and aeroelasticity. Progress in Aerospace Sciences, 46:284-327, 2010.

${ }^{3}$ Ruben B.R. Vandenheede, Luis P. Bernal, Christian L. Morrison, and Sean Humbert. Force generation of bio-inspired hover kinematics. AIAA 2012-0708, 2012.

${ }^{4}$ Alexander P. Willmott and Charles P. Ellingtion. The mechanics of flight in the hawkmoth manduca sexta. The Journal of Experimental Biology, 200:2705-2722, 1997. 


\section{Appendix}

\section{A. Hover motions with increased reduced frequency}

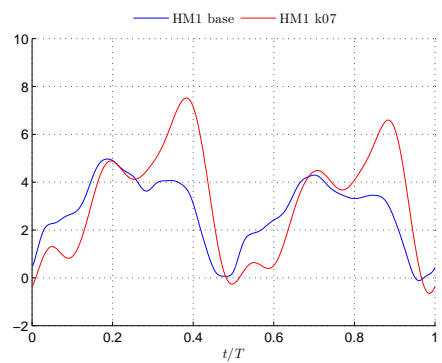

(a) Thrust coefficients HM1

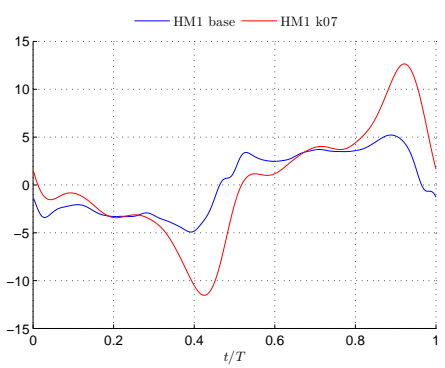

(d) Lateral force coefficients HM1

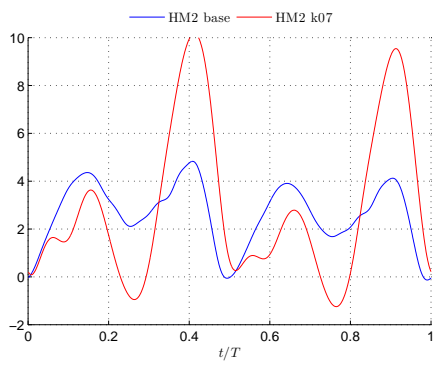

(b) Thrust coefficients HM2

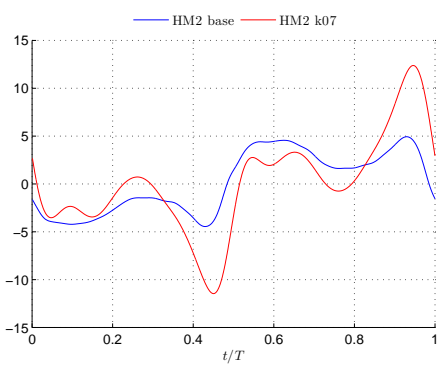

(e) Lateral force coefficients HM2

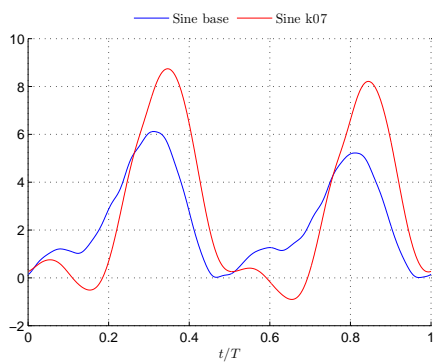

(c) Thrust coefficients Sine

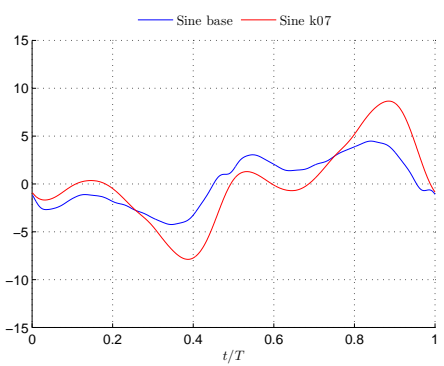

(f) Lateral force coefficients Sine

Figure 17: Hover motion with varying reduced frequency force coefficients

\section{B. Flow Visualization}

The dye flow visualization produces diffused images that can be hard to interpret therefore sketches of the most prominent flow features have been used in this report. Below a comparison of the original still images and the sketches is provided. 


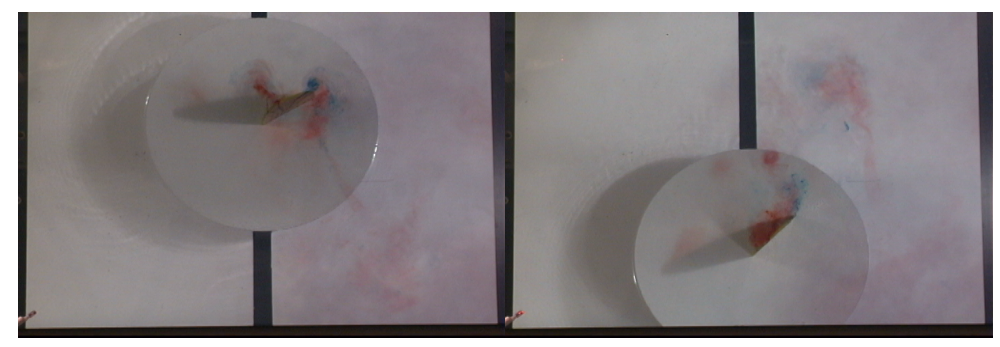

(a) Still image at $0.14 t^{*}$

(b) Still image at $0.42 t^{*}$
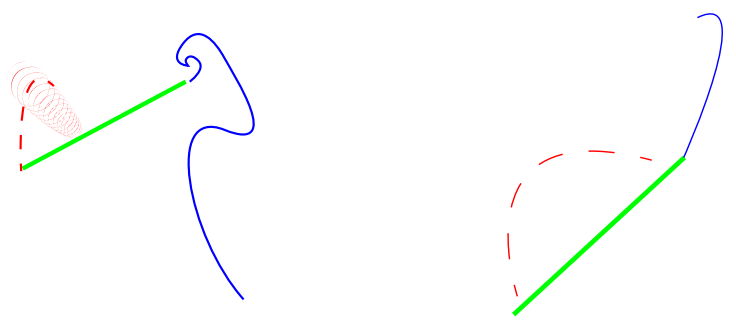

(c) Sketch of the most prominent flow(d) Sketch of the most prominent flow features at $0.14 t^{*} \quad$ features at $0.42 t^{*}$

Figure 18: Comparison of the still images and the sketches of the flow visualization features HM1 with a $10 \% T$ delayed rotation

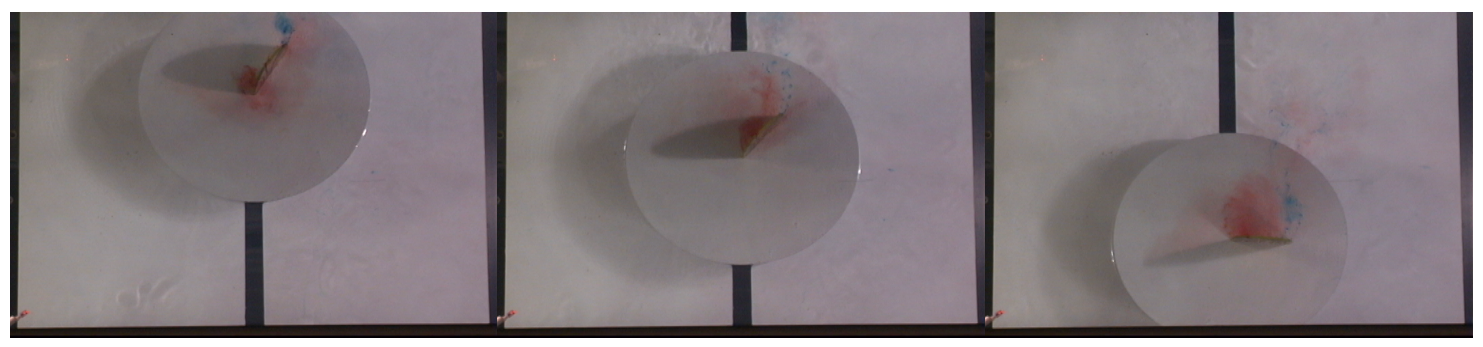

(a) Still image at $0.07 t^{*}$

(b) Still image at $0.2 t^{*}$

(c) Still image at $0.38 t^{*}$
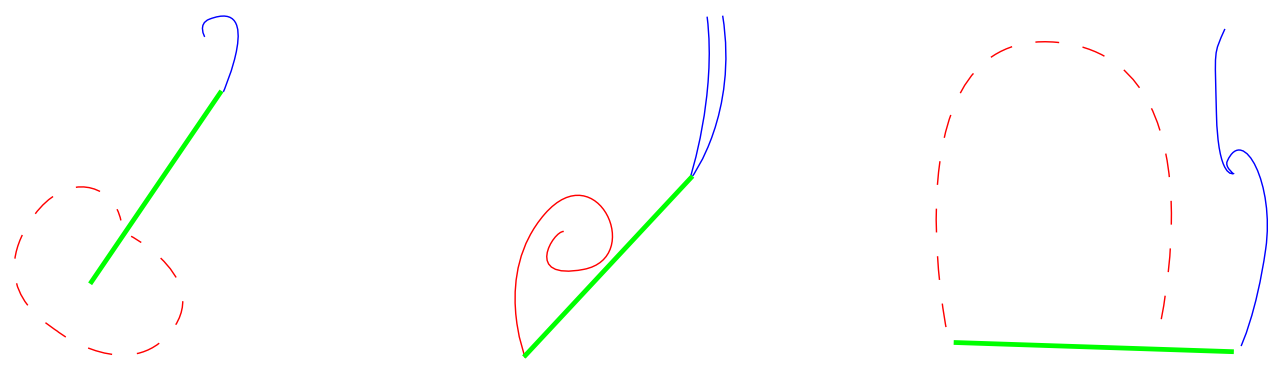

(d) Sketch of the most prominent flow(e) Sketch of the most prominent flow(f) Sketch of the most prominent flow features at $0.07 t^{*}$ features at $0.2 t^{*}$ features at $0.38 t^{*}$

Figure 19: Comparison of the still images and the sketches of the flow visualization features HM1 with a $10 \% T$ advanced rotation 


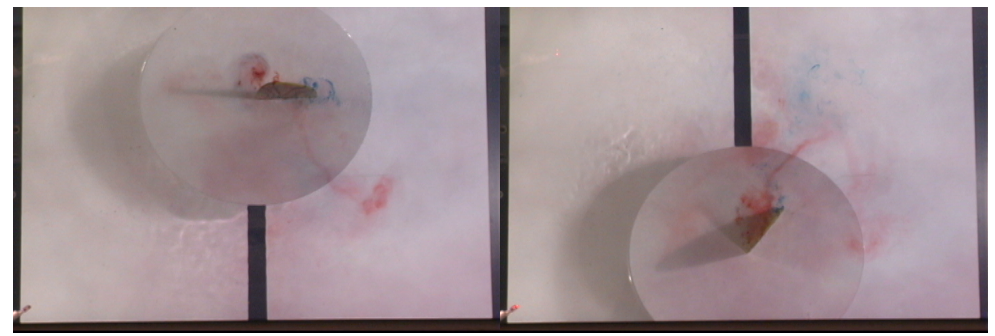

(a) Still image at $0.11 t^{*}$

(b) Still image at $0.43 t^{*}$
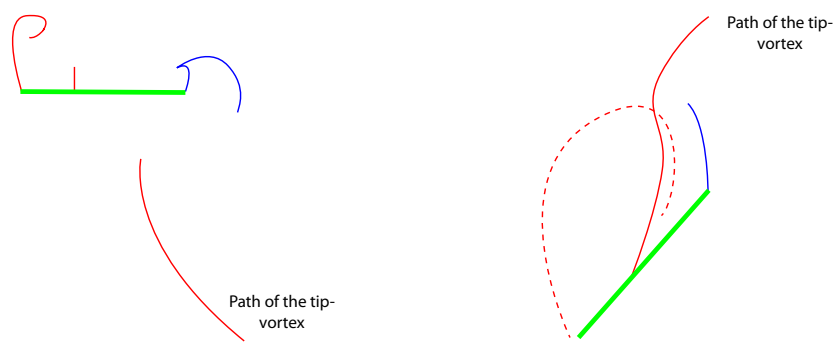

(c) Sketch of the most prominent flow(d) Sketch of the most prominent flow features at $0.11 t^{*}$ features at $0.43 t^{*}$

Figure 20: Comparison of the still images and the sketches of the flow visualization features sinusoidal motion with a $10 \% T$ delayed rotation

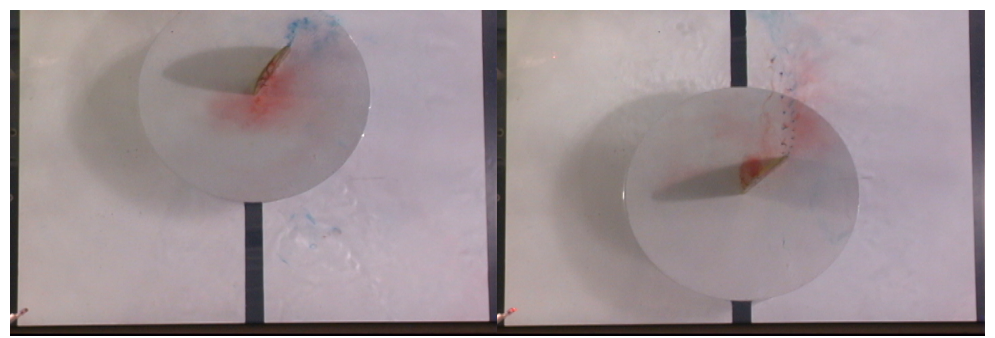

(a) Still image at $0.1 t^{*}$

(b) Still image at $0.3 t^{*}$
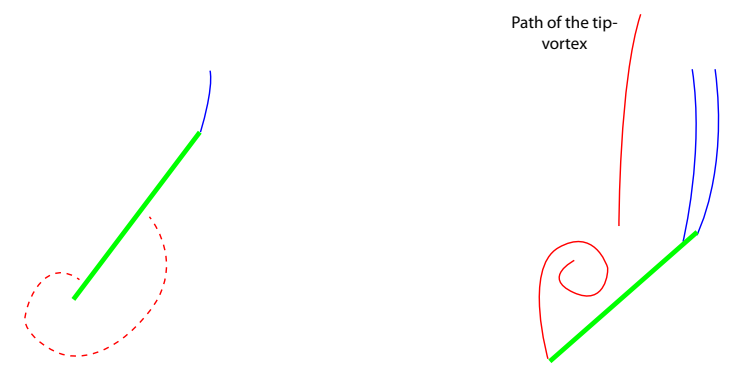

(c) Sketch of the most prominent flow(d) Sketch of the most prominent flow features at $0.1 t^{*}$ features at $0.3 t^{*}$

Figure 21: Comparison of the still images and the sketches of the flow visualization features sinusoidal motion with a $10 \% T$ delayed rotation 


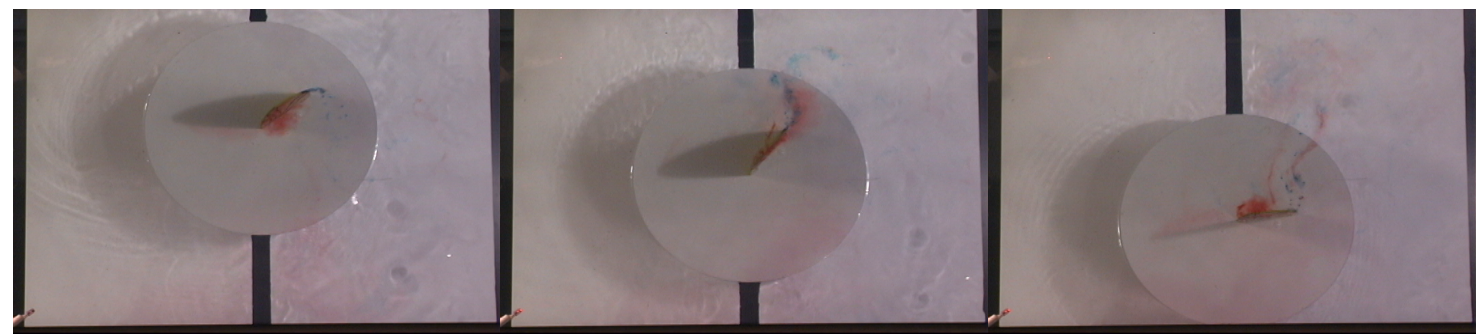

(a) Still image at $0.05 t^{*}$

(b) Still image at $0.2 t^{*}$

(c) Still image at $0.46 t^{*}$
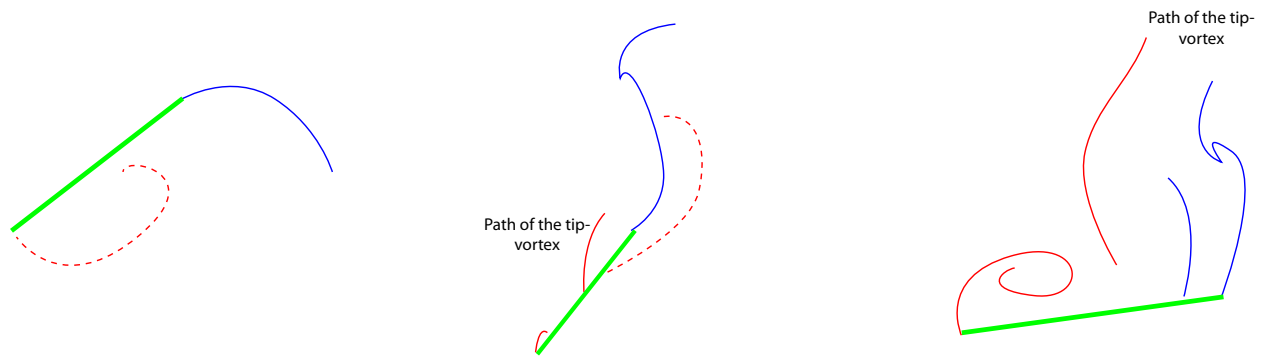

(d) Sketch of the most prominent flow(e) Sketch of the most prominent flow(f) Sketch of the most prominent flow features at $0.05 t^{*}$ features at $0.2 t^{*}$ features at $0.46 t^{*}$

Figure 22: Comparison of the still images and the sketches of the flow visualization features HM1 with a reduced frequency of 0.7

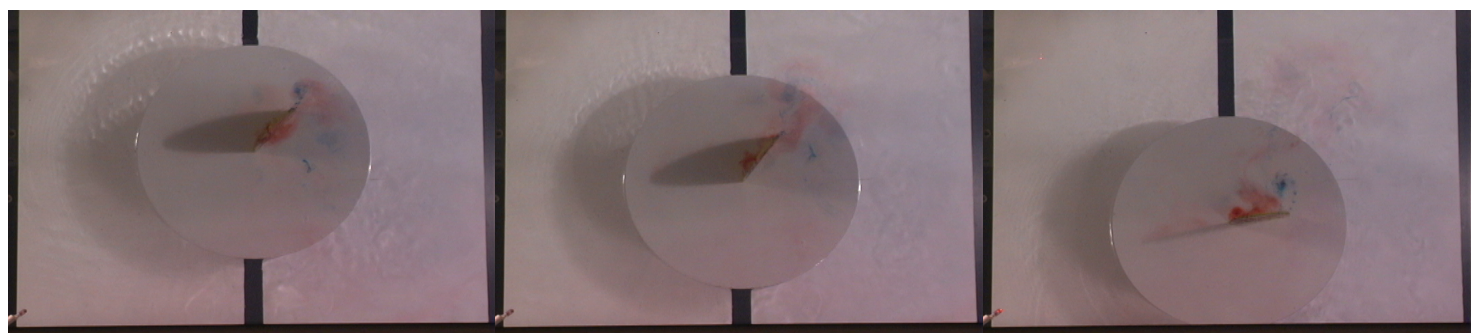

(a) Still image at $0.16 t^{*}$

(b) Still image at $0.26 t^{*}$

(c) Still image at $0.5 t^{*}$
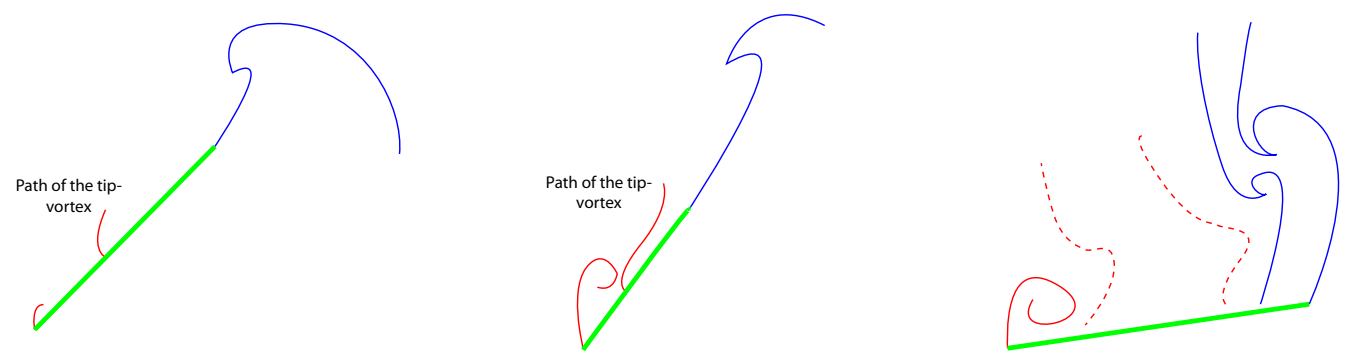

(d) Sketch of the most prominent flow(e) Sketch of the most prominent flow(f) Sketch of the most prominent flow features at $0.16 t^{*}$ features at $0.26 t^{*}$ features at $0.5 t^{*}$

Figure 23: Comparison of the still images and the sketches of the flow visualization features HM2 with a reduced frequency of 0.7 


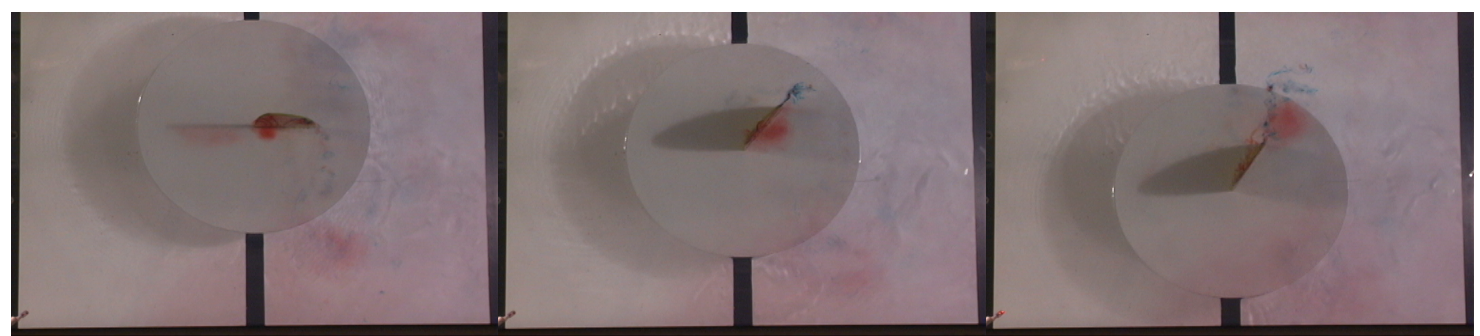

(a) Still image at $0.0 t^{*}$

(b) Still image at $0.15 t^{*}$

(c) Still image at $0.3 t^{*}$
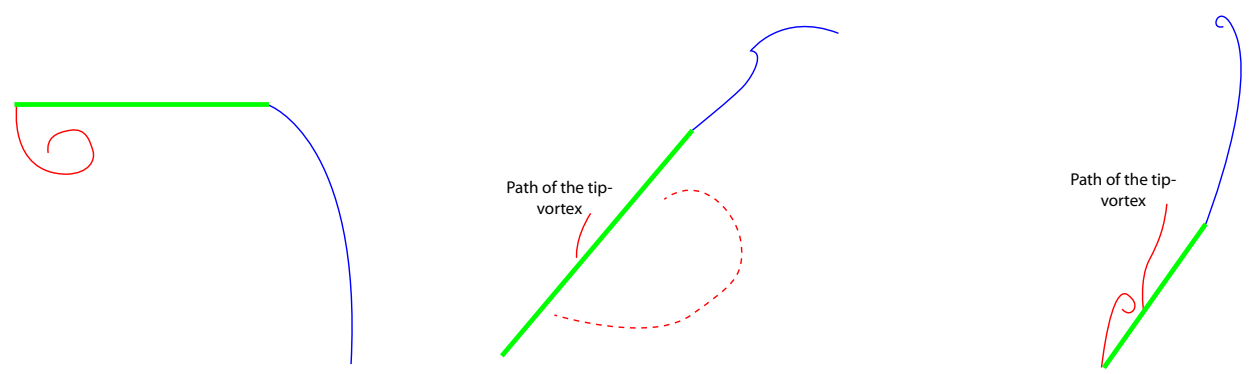

(d) Sketch of the most prominent flow(e) Sketch of the most prominent flow(f) Sketch of the most prominent flow features at $0.0 t^{*}$ features at $0.15 t^{*}$ features at $0.3 t^{*}$

Figure 24: Comparison of the still images and the sketches of the flow visualization features sine with a reduced frequency of 0.7 